\title{
Influência das características familiares no tempo de ecrã em crianças até aos 18 meses de idade
}

Cátia Palha, ${ }^{1}$ Bruna Tavares, ${ }^{2}$ Daniela Lopes Morgado, ${ }^{3}$ Débora Fonseca, ${ }^{4}$ Juliana Castro, ${ }^{5}$ Pedro Sousa Castro ${ }^{6}$

\section{RESUMO}

Objetivo: Descrever as características familiares associadas a tempo de ecrã (TE) nas crianças com idades entre 0 e 18 meses. Tipo de estudo: Estudo observacional transversal analítico.

Local: USF Além D'Ouro, USF Camélias, USF Novos Rumos, USF Terras de Santa Maria, USF Vale do Vouga e UCSP de Crestuma. População: Crianças com idade entre 0 e 18 meses, inscritas nas unidades referidas.

Métodos: Foi desenvolvido pelos autores um questionário sobre o TE das crianças e uma breve caracterização da família, de resposta voluntária e confidencial. O tratamento estatístico dos dados foi realizado através do programa Statistical Package for the Social Sciences - SPSS ${ }^{\circledR}$. Considerou-se existir significância estatística para valores de $p<0,05$.

Resultados: Foram devolvidos 142 questionários preenchidos (taxa de resposta de 42,5\%) e incluídos 137. A mediana de idades foi de 12 meses e 50,4\% eram do género masculino. A média de idades do pai foi de 34 anos e a da mãe de 33 . Cerca de $83 \%$ das crianças pertencia a famílias nucleares e 31,4\% ficava ao cuidado dos avós ou no infantário. Das 137 crianças analisadas, $81 \%$ teve exposição e, destas, mais de metade teve o primeiro contacto até aos seis meses de vida. Não se verificou diferença na duração de TE durante a semana e o fim-de-semana (71,2\% e 73\% das crianças com exposição, respetivamente). Verificou-se uma correlação estatisticamente significativa entre o TE e as variáveis «idade da criança» e «idade da mãe», sendo que crianças de mães com idade igual ou superior a 39 anos foram expostas a TE mais tardiamente.

Conclusão: Este estudo demonstrou hábitos de TE inadequados face às recomendações atuais para a idade, resultados concordantes com estudos nacionais e internacionais. A elaboração de estratégias de intervenção generalizadas, nomeadamente a capacitação dos cuidadores acerca do TE adequado à idade da criança, deve ser incentivada.

Palavras-chave: Tempo de ecrã; Crianças; Família.

\section{INTRODUÇÃO}



tempo de ecrã (TE) é definido como o tempo despendido em frente a qualquer tipo de ecrã, nomeadamente televisão, telemóveis, tablets, computador ou videojogos. ${ }^{1}$ As fontes de media são anunciadas como ferramentas educacionais na infância, ${ }^{2-3}$ perspetiva amplamente adotada pelos pais hoje em dia. ${ }^{4-5}$

Nos últimos anos, vários estudos têm sido realizados com o intuito de avaliar potenciais riscos e benefícios do TE na idade pediátrica. ${ }^{6-28}$

Por um lado, sabe-se que os riscos associados à utilização dos media incluem efeitos negativos no sono, ${ }^{6-7}$ atenção, ${ }^{8}$ aprendizagem, ${ }^{9}$ desenvolvimento da cognição, ${ }^{10-12}$ linguagem ${ }^{10,13-16}$ e capacidades socioemocionais, ${ }^{17-18}$ bem como uma maior incidência de obesidade $^{7,19-21}$ e de síndroma metabólico. ${ }^{22-24}$ Sabe-se ainda que os comportamentos sedentários praticados durante a

\footnotetext{
1. Médica Interna de Medicina Geral e Familiar. USF Camélias, ACeS Gaia.

2. Médica Interna de Medicina Geral e Familiar. USF Vale do Vouga, ACeS Entre Douro e Vouga II.

3. Médica Interna de Medicina Geral e Familiar. USF Novos Rumos, ACeS Alto Ave. 4. Médica Interna de Medicina Geral e Familiar. USF Além D'Ouro, ACeS Espinho/Gaia. 5. Médica Interna de Medicina Geral e Familiar. USF Terras de Santa Maria, ACeS Entre Douro e Vouga I.

6. Médico Interno de Medicina Geral e Familiar. UCSP de Crestuma, ACeS Espinho/Gaia.
} 
infância, incluindo o TE, parecem manter-se durante a adolescência e idade adulta. ${ }^{25-27}$

Por outro lado, apesar de o uso dos media poder ser benéfico em determinadas faixas etárias pediátricas, ${ }^{28}$ a evidência de benefício do seu uso em crianças com menos de dois anos de idade é limitada. ${ }^{11,28-29}$ Dada a imaturidade das suas capacidades simbólicas, de memória e de atenção, as crianças com idade inferior a dois anos não conseguem transferir a informação bidimensional (2D) dos media para a realidade tridimensional (3D).$^{30}$ Em relação à televisão, sabe-se que estas crianças prestam alguma atenção, mas têm uma menor compreensão face a situações equivalentes na vida real, não conseguindo compreender a maioria dos programas televisivos até cerca dos 30 meses de idade. ${ }^{11} \mathrm{Nes}-$ te sentido, a exploração prática e a interação social com os cuidadores revelam-se mais eficazes para o desenvolvimento da cognição, linguagem, motricidade e capacidades socioemocionais. ${ }^{8,31}$

Tendo em conta as conclusões dos estudos relativos às consequências do TE na idade pediátrica, algumas sociedades têm avançado com novas recomendações. Na revisão de 2016 das recomendações da Academia Americana de Pediatria, o TE, excluindo a videochamada, é desaconselhado em crianças com idade inferior a 18 meses. Entre os 18 e os 24 meses de idade, apesar de os media continuarem desaconselhados, os pais que os pretendam introduzir devem fazê-lo apenas através de programas/aplicações de alta qualidade, acompanhando sempre as crianças no seu uso. É ainda recomendada a não utilização de ecrãs durante as refeições e uma hora antes de deitar. ${ }^{28}$ Mais recentemente, a Sociedade Canadiana de Pediatria reforçou as recomendações de evicção dos media em crianças com menos de 24 meses de idade. ${ }^{1}$

Apesar destas recomendações, vários estudos revelaram que o TE é superior ao recomendado. Ao nível da população portuguesa, um estudo de 2013 concluiu que em $80,9 \%$ das crianças a televisão tinha sido introduzida na sua rotina antes dos dois anos de idade. ${ }^{32}$

Considerando que a maior parte do TE ocorre em casa, ${ }^{33}$ os pais encontram-se numa posição privilegiada de influência nestes hábitos. Vários estudos avaliaram potenciais fatores determinantes do TE na idade pediátrica, tendo sido encontradas algumas associações com o local de residência (urbano versus rural), ${ }^{7,34}$ condição socioeconómica, , $527,29,34-37$ estrutura familiar, ${ }^{38}$ escolaridade parental, ${ }^{5,27,29,34-35,39-41}$ nacionalidade parental, ${ }^{34}$ hábitos alimentares, ${ }^{7,42}$ presença de televisão no quarto, ${ }^{34-35,41,43-44}$ frequência de infantário, ${ }^{5,27,39}$ idade da criança, ${ }^{5,27,36,39-40,43,45}$ entre outros.

Dada a importância do cumprimento das recomendações atuais relativas ao TE na idade pediátrica e a escassez de estudos na população portuguesa, em particular nas crianças pequenas, os autores consideraram relevante investigar os fatores associados à exposição precoce a ecrãs. O objetivo geral deste estudo foi descrever as características familiares associadas ao TE nas crianças com idades entre 0 e 18 meses. Pretendeu-se caracterizar as famílias quanto à sua estrutura, características demográficas e socioeconómicas, bem como os hábitos de consumo de TE destas crianças, averiguando possíveis associações.

\section{MÉTODOS}

Foi realizado um estudo observacional transversal e analítico na Unidade de Saúde Familiar (USF) Além D'Ouro, USF Camélias, USF Novos Rumos, USF Terras de Santa Maria, USF Vale do Vouga e na Unidade de Cuidados de Saúde Personalizados (UCSP) de Crestuma, que correspondem às unidades de saúde (US) dos autores. O trabalho foi iniciado em junho de $2017 \mathrm{com}$ a elaboração do protocolo de investigação. Este foi aprovado pelos coordenadores de cada US, pelos conselhos clínicos dos Agrupamentos de Centros de Saúde (ACeS) de Gaia, Espinho/Gaia, Alto Ave, Entre Douro e Vouga I e Entre Douro e Vouga II e pela Comissão de Ética da Administração Regional de Saúde do Norte (ARSN) até junho de 2018.

Os investigadores elaboraram um questionário que foi submetido a um processo de cognitive debriefing, de modo a avaliar o nível de compreensão do mesmo pela população alvo. Cada questionário apresentava um texto introdutório em que se explicava o objetivo principal do estudo e se solicitava a participação voluntária, assegurando ainda a confidencialidade no tratamento de todos os dados recolhidos. As questões serviram para avaliar o TE das crianças e fazer uma breve caracterização da família.

Foram identificadas 1.000 crianças, número correspondente ao total de crianças com idades compreendidas entre 0 e 18 meses a 1 de junho de 2017 no total das 
seis US (A, B, C, D, E e F). Para estimar o tamanho amostral foi utilizado o sítio da internet www.vsai.pt/amostragem.php para uma prevalência esperada de TE superior ao recomendado de $50 \%$ (prevalência estimada de evento desconhecido), uma precisão de 0,05 e um intervalo de confiança de $95 \%$. Ao número obtido, de 278 crianças, adicionou-se $20 \%$ de forma a minimizar as perdas esperadas por falta de adesão/resposta aos questionários enviados, perfazendo um total de 334 crianças.

À data de 25 de maio de 2018 foram obtidas as listagens das crianças inscritas nas seis US que tinham idades compreendidas entre 0 e 18 meses. $O$ número de crianças selecionadas foi ponderado de acordo com o número total de crianças por US (US A com 15,6\% das crianças, US B com $34,5 \%$, US C com $5,9 \%$, US D com $13 \%$, US E com $15,5 \%$ e US F com $15,5 \%$ ), correspondendo a 52 crianças da US A, 115 da US B, 20 da US C, 43 da US D, 52 da US E e 52 da US F. Os questionários foram enviados a 1 de junho de 2018, por carta, para a morada associada ao processo clínico das crianças selecionadas de forma aleatória, através do sítio da internet www.random.org. Foi colocada uma caixa identificada em cada US participante para os pais das crianças devolverem os questionários até ao dia 31 de agosto de 2018.

Os critérios de exclusão foram os seguintes: crianças pertencentes a um agregado familiar selecionadas previamente e crianças cujo questionário não tinha resposta nos pontos 1.2, 3.1 e sem resposta no ponto 3.2, após resposta positiva no ponto 3.1.

O tratamento estatístico dos dados foi realizado com recurso ao programa Statistical Package for the Social Sciences-SPSS ${ }^{\circledast}$. Para a análise descritiva das variáveis numéricas foi realizado um teste de normalidade (teste de Kilmogorov-Smirnov com correção de Lilliefors e Shapiro-Wilk) e posteriormente aferidas as medidas de localização [mínimo e máximo, média e desvio-padrão (DP) para variáveis normais, simétricas, e mediana e amplitude interquartil para variáveis não normais ou assimétricas]. Para a descrição das variáveis categóricas foram analisadas as frequências relativas e absolutas de cada categoria.

Através do teste de Qui-quadrado de Pearson foi estudada a relação entre TE e as seguintes variáveis: «escolaridade do pai e da mãe», «principal fonte de rendimento», «tipo de família», «quem toma conta da criança na maior parte do dia», «ter televisão no quarto» e «ter

\begin{tabular}{|c|c|c|c|}
\hline \multicolumn{4}{|c|}{ QUADRO I. Distribuição total das crianças por idades } \\
\hline $\begin{array}{l}\text { Idade } \\
\text { (meses) }\end{array}$ & $\begin{array}{c}\text { Frequência } \\
\text { absoluta }\end{array}$ & $\begin{array}{l}\text { Frequência } \\
\text { relativa }\end{array}$ & $\begin{array}{l}\text { Frequência } \\
\text { acumulada }\end{array}$ \\
\hline 0 & 1 & 0,7 & 0,7 \\
\hline 1 & 3 & 2,2 & 2,9 \\
\hline 2 & 1 & 0,7 & 3,6 \\
\hline 3 & 4 & 2,9 & 6,6 \\
\hline 4 & 9 & 6,6 & 13,1 \\
\hline 5 & 5 & 3,6 & 16,8 \\
\hline 6 & 10 & 7,3 & 24,1 \\
\hline 7 & 6 & 4,4 & 28,5 \\
\hline 8 & 4 & 2,9 & 31,4 \\
\hline 9 & 13 & 9,5 & 40,9 \\
\hline 10 & 9 & 6,6 & 47,4 \\
\hline 11 & 2 & 1,5 & 48,9 \\
\hline 12 & 9 & 6,6 & 55,5 \\
\hline 13 & 6 & 4,4 & 59,9 \\
\hline 14 & 5 & 3,6 & 63,5 \\
\hline 15 & 13 & 9,5 & 73,0 \\
\hline 16 & 9 & 6,6 & 79,6 \\
\hline 17 & 7 & 5,1 & 84,7 \\
\hline 18 & 21 & 15,3 & 100,0 \\
\hline Total & 137 & 100,0 & \\
\hline
\end{tabular}

informação sobre TE». Com base no teste $U$ de MannWhitney foi estudada a correlação entre TE e a «idade da criança», «idade do pai», «idade da mãe»e «número de elementos do agregado familiar» e a correlação entre «idade do primeiro contacto com TE» e a «idade da mãe».

Considerou-se existir significância estatística para valores de $p<0,05$.

\section{RESULTADOS}

\section{Amostra}

Foram devolvidos 142 questionários preenchidos, perfazendo uma taxa de resposta global de $42,5 \%$ (15,4\%, $17,3 \%, 43,5 \%, 50,0 \%, 63,5 \%$ e $74,4 \%$ para a US A, E, B, C, F e D, respetivamente). Foram excluídos cinco questionários por resposta afirmativa à pergunta 3.1 - «desde que nasceu, a criança teve algum $\mathrm{TE}$ », sem resposta à pergunta 3.2 - «que idade tinha a criança quando teve pela 
primeira vez TE». Foram, portanto, analisados 137 questionários.

\section{Caracterização das crianças e famílias Idade e sexo das crianças}

A mediana de idades das crianças foi de 12 meses (percentil 25/P25=7; percentil 75/P75=16). Relativamente ao sexo, 50,4\% ( $n=69)$ era do sexo masculino e 49,6\% ( $n=68)$ era do sexo feminino. O Quadro I apresenta a distribuição das crianças por idade.

\section{Pais das crianças}

A média de idades do pai das crianças foi de 34 anos (DP=6). A mediana de idades da mãe das crianças foi de 33 anos (P25=30; $\mathrm{P} 75=37$ ). Verificou-se que a maioria dos pais e das mães das crianças estudadas tinha 12 anos de escolaridade ou ensino superior. A maioria dos pais era de nacionalidade portuguesa. Obteve-se que a fonte principal de rendimento foi o trabalho $(91,3 \%, n=125)$. A caracterização dos pais quanto à escolaridade, nacionalidade e fonte de rendimento encontra-se no Quadro II.

\section{Famílias}

Relativamente às famílias verificou-se que a maioria das crianças estudadas pertencia a uma família nuclear $(82,5 \%$, $n=113$ ). O número de elementos do agregado familiar que mais se verificou foi de quatro (P25=3; P75=4). Uma percentagem semelhante foi encontrada em relação a ter ou não irmãos (sim: 48,9\%, $n=67$; não: $51,1 \%, n=70)$. Em relação à pessoa/instituição responsável por tomar conta da criança na maioria do tempo obteve-se que os avós e o infantário/creche foram os mais frequentes $(31,4 \%, n=43$ cada), seguido dos pais $(28,5 \%, n=39)$. A caracterização das famílias encontra-se no Quadro III.

\section{Informação sobre tempo de ecrã}

Em 137 questionários analisados foi encontrado um questionário sem resposta à pergunta 3.3 - «Alguma
QUADRO II. Escolaridade, nacionalidade e fonte de rendimento dos pais das crianças

\begin{tabular}{|c|c|c|c|}
\hline & $\begin{array}{l}\text { Frequência } \\
\text { absoluta }\end{array}$ & $\begin{array}{c}\text { Frequência } \\
\text { relativa }\end{array}$ & $\begin{array}{l}\text { Frequência } \\
\text { acumulada }\end{array}$ \\
\hline \multicolumn{4}{|l|}{ Escolaridade do pai } \\
\hline Menos de 4 anos de escolaridade & 2 & 1,5 & 1,5 \\
\hline 4 Anos de escolaridade & 1 & 0,7 & 2,2 \\
\hline 6 Anos de escolaridade & 19 & 13,9 & 16,1 \\
\hline 9 Anos de escolaridade & 31 & 22,6 & 38,7 \\
\hline 12 Anos de escolaridade & 40 & 29,2 & 67,9 \\
\hline Curso tecnológico/profissional & 2 & 1,5 & 69,4 \\
\hline Ensino superior & 42 & 30,6 & 100 \\
\hline Total & 137 & 100,0 & \\
\hline \multicolumn{4}{|l|}{ Escolaridade da mãe } \\
\hline 4 Anos de escolaridade & 4 & 2,9 & 2,9 \\
\hline 6 Anos de escolaridade & 4 & 2,9 & 5,8 \\
\hline 9 Anos de escolaridade & 22 & 16,1 & 21,9 \\
\hline 12 Anos de escolaridade & 41 & 29,9 & 51,8 \\
\hline Curso tecnológico/profissional & 6 & 4,4 & 56,2 \\
\hline Ensino superior & 60 & 43,8 & 100,0 \\
\hline Total & 137 & 100,0 & \\
\hline \multicolumn{4}{|l|}{ Nacionalidade do pai } \\
\hline Portuguesa & 134 & 97,8 & 97,8 \\
\hline Outra & 3 & 2,2 & 100,0 \\
\hline Total & 137 & 100,0 & \\
\hline \multicolumn{4}{|l|}{ Nacionalidade da mãe } \\
\hline Portuguesa & 133 & 97,1 & 97,1 \\
\hline Outra & 4 & 2,9 & 100,0 \\
\hline Total & 137 & 100,0 & \\
\hline \multicolumn{4}{|l|}{ Fonte de rendimento } \\
\hline A cargo da família & 1 & 0,7 & 0,7 \\
\hline Outro subsídio temporário & 4 & 29 & 3.6 \\
\hline Rendimento social de inserção & $\begin{array}{l}4 \\
4\end{array}$ & $\begin{array}{l}2,9 \\
2,9\end{array}$ & $\begin{array}{l}3,0 \\
6,5\end{array}$ \\
\hline Subsídio de desemprego & 3 & 2,2 & 8,7 \\
\hline Trabalho & 125 & 91,3 & 100,0 \\
\hline Total & 137 & 100,0 & \\
\hline
\end{tabular}

vez foi informado sobre o TE adequado à idade da criança». Dos 136 com resposta, em 73,5\% $(n=100)$ a resposta foi negativa e em $26,5 \%$ ( $n=36$ ) foi afirmativa.

Em relação aos questionários cuja resposta relativa à informação sobre o TE foi positiva, a mediana do nú- 


\begin{tabular}{|c|c|c|c|}
\hline & $\begin{array}{l}\text { Frequência } \\
\text { absoluta }\end{array}$ & $\begin{array}{l}\text { Frequência } \\
\text { relativa }\end{array}$ & $\begin{array}{l}\text { Frequência } \\
\text { acumulada }\end{array}$ \\
\hline \multicolumn{4}{|l|}{ Tipo de família } \\
\hline Alargada & 17 & 12,4 & 12,4 \\
\hline Monoparental & 5 & 3,6 & 16,1 \\
\hline Nuclear & 113 & 82,5 & 98,5 \\
\hline Numerosa & 1 & 0,7 & 99,3 \\
\hline Reconstituída & 1 & 0,7 & 100,0 \\
\hline Total & 137 & 100,0 & \\
\hline \multicolumn{4}{|l|}{$\begin{array}{l}\text { Número de elementos do } \\
\text { agregado }\end{array}$} \\
\hline 2 Elementos & 3 & 2,2 & 2,2 \\
\hline 3 Elementos & 56 & 40,9 & 43,1 \\
\hline 4 Elementos & 54 & 39,4 & 82,5 \\
\hline 5 Elementos & 17 & 12,4 & 94,9 \\
\hline 6 Elementos & 6 & 4,4 & 99,3 \\
\hline 8 Elementos & 1 & 0,7 & 100,0 \\
\hline Total & 137 & 100,0 & \\
\hline \multicolumn{4}{|l|}{ Ter irmãos } \\
\hline Não & 67 & 48,9 & 48,9 \\
\hline Sim & 70 & 51,1 & 100,0 \\
\hline Total & 137 & 100,0 & \\
\hline \multicolumn{4}{|l|}{ Quem toma conta da criança } \\
\hline Ama (com mais crianças) & 6 & 4,4 & 4,4 \\
\hline Ama (sem mais crianças) & 5 & 3,6 & 8,0 \\
\hline Avós & 43 & 31,4 & 39,4 \\
\hline Infantário/Creche & 43 & 31,4 & 70,8 \\
\hline Outro & 1 & 0,7 & 71,5 \\
\hline Pais & 39 & 28,5 & 100,0 \\
\hline Total & 137 & 100,0 & \\
\hline
\end{tabular}

19\% ( $n=26$ ) não teve. O Quadro IV explicita a distribuição por idades das crianças com e sem TE à data de resposta do questionário. Verificou-se que a maioria das crianças com TE teve o primeiro contacto nos primeiros meses de vida, sendo que mais de metade delas foi exposta até aos seis meses (Quadro V).

\section{Tempo de ecrã: quando e quanto?}

Foram analisadas quantas horas por dia as crianças estavam expostas a ecrãs. Verificou-se que durante a semana a maioria das crianças tinha menos de uma hora de TE $(71,2 \%, n=79)$ e durante o fim-de-semana a mesma quantidade de horas $(73 \%$, $n=81)$. Durante as principais refeições, $25,5 \%(n=36)$ das crianças tinha acesso a TE. A distribuição do TE encontra-se no Quadro VI.

Relativamente a ter televisão no quarto, dos 126 questionários com resposta válida a esta pergunta (11 omissos) em $61,9 \%$ $(n=78)$ a resposta foi negativa e em $38,1 \%$ $(n=48)$ a resposta foi afirmativa.

\section{Correlações com o tempo de ecrã}

Foi estudado se o ter ou não TE se correlacionava com as restantes variáveis de caracterização socioeconómica e familiar.

Através do teste Ude Mann-Whitneyverificou-se que o grupo de crianças com TE tinha uma idade superior (mediana de 12 meses) à do grupo das crianças sem $\mathrm{TE}$ (mediana de seis meses), variando de for-

mero de fontes de informação foi de uma fonte (mínimo 1; máximo 5). Obteve-se um total de 61 fontes, sendo que a mais frequentemente identificada foi o médico/enfermeiro $(34,4 \%, n=22)$, seguido da Internet $(24,6 \%, n=15)$.

\section{Caracterização dos hábitos de consumo de tempo de ecrã}

\section{Ter ou não tempo de ecrã}

Das 137 crianças analisadas, $81 \%(n=111)$ teve TE e ma estatisticamente significativa $(p=0,006)$.

Através do teste de Qui-quadrado de Pearson obteve-se que a variável TE não se correlacionou de forma significativa com o sexo da criança $\left(X^{2}=0,647 ; p=0,421\right)$.

Através do teste $U$ de Mann-Whitney verificou-se que as variáveis «idade do pai»e «idade da mãe» não variaram de forma significativa consoante se criança tinha ou não tinha TE ( $p=0,285 ; p=0,920$, respetivamente). Para correlacionar a variável «idade do primeiro contacto com TE» das crianças analisadas com a idade dos progenitores foi feita a divisão dos indivíduos se- 


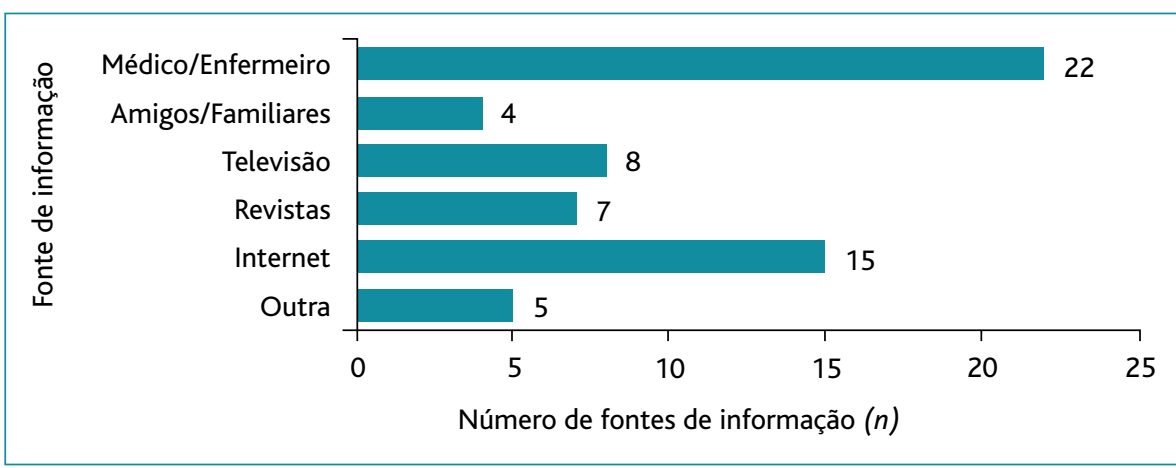

Figura 1. Fonte de informação sobre o tempo de ecrã.

gundo a idade da mãe/pai em quatro grupos. Para a idade do pai foram considerados os seguintes: idades inferiores ou iguais a 28 ; idades entre 29 e 34 ; idades en- tre 35 e 40 e idade igual ou superior a 41 anos. Para a idade da mãe foram considerados os seguintes grupos: idades inferiores ou iguais a 28 ; idades entre 29 e 33; idades entre 34 e 38 e idade igual ou superior a 39 anos. Os grupos foram realizados tendo em conta a média e o DP (pai: média $=34,28$; $\mathrm{DP}=5,99$; mãe: média $=32,99$; $\mathrm{DP}=5,22)$. Foi realizado o teste Kruskal Wallis para verificar diferenças entre grupos, sendo que para a idade do pai não se verificaram diferenças entre os grupos $(\mathrm{R}=4,406$; $p=0,221$ ). Para a idade da mãe verificou-se diferença

\begin{tabular}{|c|c|c|c|c|c|c|c|}
\hline \multirow{2}{*}{$\begin{array}{l}\text { Idade } \\
\text { (meses) }\end{array}$} & \multicolumn{3}{|c|}{ Crianças com tempo de ecrã } & \multicolumn{3}{|c|}{ Crianças sem tempo de ecrã } & \multirow[b]{2}{*}{ Total } \\
\hline & $\begin{array}{c}\text { Frequência } \\
\text { absoluta }\end{array}$ & $\begin{array}{c}\text { Frequência } \\
\text { relativa }\end{array}$ & $\begin{array}{l}\text { Frequência } \\
\text { acumulada }\end{array}$ & $\begin{array}{c}\text { Frequência } \\
\text { absoluta }\end{array}$ & $\begin{array}{l}\text { Frequência } \\
\text { relativa }\end{array}$ & $\begin{array}{l}\text { Frequência } \\
\text { acumulada }\end{array}$ & \\
\hline 0 & 0 & 0 & 0 & 1 & 3,8 & 3,8 & 1 \\
\hline 1 & 1 & 0,9 & 0,9 & 2 & 7,7 & 11,5 & 3 \\
\hline 2 & 0 & 0,0 & 0,9 & 1 & 3,8 & 15,3 & 1 \\
\hline 3 & 2 & 1,8 & 2,7 & 2 & 7,7 & 23,0 & 4 \\
\hline 4 & 5 & 4,5 & 7,2 & 4 & 15,4 & 38,4 & 9 \\
\hline 5 & 4 & 3,6 & 10,8 & 1 & 3,8 & 42,3 & 5 \\
\hline 6 & 7 & 6,3 & 17,1 & 3 & 11,5 & 53,8 & 10 \\
\hline 7 & 5 & 4,5 & 21,6 & 1 & 3,8 & 57,6 & 6 \\
\hline 8 & 4 & 3,6 & 25,2 & 0 & 0,0 & 57,6 & 4 \\
\hline 9 & 12 & 10,8 & 36,0 & 1 & 3,8 & 61,5 & 13 \\
\hline 10 & 8 & 7,2 & 43,2 & 1 & 3,8 & 65,3 & 9 \\
\hline 11 & 2 & 1,8 & 45,0 & 0 & 0,0 & 65,3 & 2 \\
\hline 12 & 8 & 7,2 & 52,3 & 1 & 3,8 & 69,2 & 9 \\
\hline 13 & 6 & 5,4 & 57,7 & 0 & 0,0 & 69,2 & 6 \\
\hline 14 & 4 & 3,6 & 61,3 & 1 & 3,8 & 73,0 & 5 \\
\hline 15 & 10 & 9,0 & 70,3 & 3 & 11,5 & 84,6 & 13 \\
\hline 16 & 8 & 7,2 & 77,5 & 1 & 3,8 & 88,4 & 9 \\
\hline 17 & 7 & 6,3 & 83,8 & 0 & 0,0 & 88,4 & 7 \\
\hline 18 & 18 & 16,2 & 100,0 & 3 & 11,5 & 100,0 & 21 \\
\hline Total & 111 & 100,0 & & 26 & 100,0 & & \\
\hline
\end{tabular}




\begin{tabular}{|c|c|c|c|}
\hline $\begin{array}{l}\text { Idade } \\
\text { (meses) }\end{array}$ & $\begin{array}{l}\text { Frequência } \\
\text { absoluta }\end{array}$ & $\begin{array}{c}\text { Frequência } \\
\text { relativa }\end{array}$ & $\begin{array}{l}\text { Frequência } \\
\text { acumulada }\end{array}$ \\
\hline 0 & 1 & 0,9 & 0,9 \\
\hline 1 & 2 & 1,8 & 2,7 \\
\hline 2 & 7 & 6,3 & 9 \\
\hline 3 & 9 & 8,1 & 17,1 \\
\hline 4 & 14 & 12,6 & 29,7 \\
\hline 5 & 12 & 10,8 & 40,5 \\
\hline 6 & 23 & 20,7 & 61,3 \\
\hline 7 & 5 & 4,5 & 65,8 \\
\hline 8 & 5 & 4,5 & 70,3 \\
\hline 9 & 12 & 10,8 & 81,1 \\
\hline 10 & 2 & 1,8 & 82,9 \\
\hline 11 & 3 & 2,7 & 85,6 \\
\hline 12 & 12 & 10,8 & 96,4 \\
\hline 14 & 1 & 0,9 & 97,3 \\
\hline 15 & 1 & 0,9 & 98,2 \\
\hline 16 & 1 & 0,9 & 99,1 \\
\hline 18 & 1 & 0,9 & 100 \\
\hline Total & 111 & 100 & \\
\hline
\end{tabular}

entre grupos $(\mathrm{R}=11,357 ; p=0,010)$. Tendo em conta que se verificou diferença estatisticamente significativa para a idade da mãe realizou-se o teste $U$ de MannWhitney entre grupos, para esclarecer qual apresentava diferença significativa. $O$ resultado revela que quando a idade da mãe é igual ou superior a 39 anos as crianças foram expostas a ecrãs mais tardiamente, em comparação com as mães com idade inferiores ou iguais a 28 anos $(\mathrm{U}=88,5 ; p=0,034)$, com idades entre 29 e 33 anos $(U=193,0 ; p=0,041)$ e com idades entre 34 e 38 anos $(\mathrm{U}=216,0 ; p=0,001)$.

Foi analisado se o TE se correlacionava com a escolaridade do pai e da mãe (agrupamento de menos de 12 anos de escolaridade versus agrupamento de 12 anos de escolaridade ou curso tecnológico/profissional versus ensino superior). Verificou-se através do teste de Qui-quadrado de Pearson que esta correlação foi não significativa para ambos (pai: $X^{2}=2,598 ; p=0,273$; mãe: $\mathrm{X}^{2}=1,610 ; p=0,447$ ).
Em relação à correlação entre o TE e a fonte de rendimento (trabalho versus agrupamento de outras fontes de rendimento), esta não foi estatisticamente significativa $\left(\mathrm{X}^{2}=0,265 ; p=0,587\right)$.

Verificou-se, através do teste de Qui-quadrado de Pearson, que o TE não se correlacionava com o tipo de família (nuclear versus agrupamento dos outros tipos de família) $\left(\mathrm{X}^{2}=0,170 ; p=0,680\right)$ nem com a existência de irmãos $\left(\mathrm{X}^{2}=1,304 ; p=0,253\right)$.

Através do teste $U$ de Mann-Whitney verificou-se que o número de elementos do agregado familiar não variou de forma significativa consoante a variável TE $(p=0,752)$.

No que diz respeito à correlação entre o TE e a variável «quem toma conta da criança» (pais versus avós versus infantário/creche versus agrupamento dos restantes possíveis responsáveis), esta não foi estatisticamente significativa $\left(X^{2}=2,489 ; p=0,477\right)$. O mesmo se verificou na correlação entre o TE e ter televisão no quarto $\left(\mathrm{X}^{2}=0,065 ; p=0,798\right)$.

Relativamente à correlação entre TE e ter informação sobre TE obteve-se que não existiram diferenças significativas $\left(\mathrm{X}^{2}=2,375 ; p=0,123\right)$.

\section{DISCUSSÃO}

Este estudo demonstrou hábitos de TE inadequados face às recomendações atuais para a idade, constatando-se que $81,0 \%$ das crianças até aos 18 meses de idade já tinha sido exposta a um ecrã. Estes resultados são concordantes com um estudo português de 2013, no qual $80,9 \%$ das crianças tinha iniciado hábitos de ver televisão antes dos dois anos de idade. ${ }^{32}$

À semelhança de outros estudos internacionais, o ter ou não TE não se correlacionou significativamente com o sexo da criança, ${ }^{5,27,29,35}$ mas variou consoante a idade da criança, ${ }^{5,27,29}$ tendo também sido reportado na literatura um aumento do tempo de exposição por dia com a idade da criança. ${ }^{36,39-40,43,45}$ Neste estudo parece haver uma tendência para o início do TE em idades muito precoces, mais concretamente entre os quatro e os seis meses, idade em que habitualmente a criança se torna mais atenta aos sons, curiosa, interativa, inicia a diversificação alimentar e em que geralmente começa a ficar ao cuidado de outras pessoas além dos pais. Deste modo, aos 12 meses de idade, quase todas as crianças com TE analisadas $(96,4 \%)$ já tinham tido a primei- 
QUADRO VI. Caracterização do tempo de ecrã

\begin{tabular}{|c|c|c|c|}
\hline & $\begin{array}{l}\text { Frequência } \\
\text { absoluta }\end{array}$ & $\begin{array}{l}\text { Frequência } \\
\text { relativa }\end{array}$ & $\begin{array}{l}\text { Frequência } \\
\text { acumulada }\end{array}$ \\
\hline \multicolumn{4}{|c|}{$\begin{array}{l}\text { Tempo de ecrã durante a } \\
\text { semana }\end{array}$} \\
\hline Menos de 1 hora & 79 & 71,2 & 71,2 \\
\hline 1 a 2 horas & 26 & 23,4 & 94,6 \\
\hline Mais de 2 horas & 6 & 5,4 & 100,0 \\
\hline Total & 111 & 100 & \\
\hline \multicolumn{4}{|c|}{$\begin{array}{l}\text { Tempo de ecrã durante o } \\
\text { fim-de-semana }\end{array}$} \\
\hline Menos de 1 hora & 81 & 73,0 & 73 \\
\hline 1 a 2 horas & 22 & 19,8 & 92,8 \\
\hline Mais de 2 horas & 8 & 7,2 & 100,0 \\
\hline Total & 111 & 100 & \\
\hline \multicolumn{4}{|c|}{$\begin{array}{l}\text { Tempo de ecrã durante a } \\
\text { refeição }\end{array}$} \\
\hline Não & 75 & 54,7 & 74,5 \\
\hline Sim & 36 & 25,5 & 100 \\
\hline Total & 111 & 100 & \\
\hline
\end{tabular}

dadores para o risco de perturbação do sono e consequente impacto nas atividades diurnas. ${ }^{28}$

Em relação à idade da mãe não foi encontrada uma diferença significativa entre o ter ou não TE. Este resultado não é consensual na literatura, estando os estudos divididos entre a não associação a $\mathrm{TE}^{41}$ e a presença de uma associação negativa com a idade da mãe. ${ }^{29}$ Relativamente à idade de início de TE, crianças de mães mais velhas, nomeadamente acima dos 39 anos, iniciavam esta exposição em idades mais tardias. Uma vez que os hábitos de TE dos pais parecem refletir-se nos hábitos de TE dos filhos,,$^{29,40-41}$ os autores ponderaram uma eventual associação deste resultado com este facto. No entanto, não foram encontrados estudos que avaliassem os hábitos de TE dos pais, nem a associação entre a idade materna e a idade de início de TE nas crianças. Um estudo demonstrou que crianças de mães mais velhas tinham menor quantidade de TE por dia, embora

ra exposição. Não se constataram diferenças significativas no TE entre a semana e o fim-de-semana, tendo a maioria das crianças menos de uma hora cumulativa de exposição por dia, tempo concordante com o encontrado nalguns estudos ${ }^{40}$ e inferior a outros. ${ }^{43}$ TE superiores foram verificados em percentagens semelhantes às descritas no estudo português de 2015 (28,8\% versus $21 \%$ durante a semana; $27,0 \%$ versus $32 \%$ ao fim de semana) para crianças com menos de dois anos de idade. ${ }^{44}$

Cerca de um quarto das crianças tinha TE durante a refeição, frequência inferior à encontrada em estudos portugueses que incluíram crianças mais velhas (55$72 \%) . .^{32,44,46}$ De salientar que as recomendações da AAP desaconselham o TE durante a refeição nos primeiros cinco anos de idade. ${ }^{28}$

Embora neste estudo não tenha sido encontrada uma correlação entre o ter e não ter TE e a presença de televisão no quarto, outros estudos demonstraram uma associação positiva. ${ }^{35,43-44}$ Esta associação, aliada à recomendação atual de evitar o TE na hora antes de ir dormir, mantém a necessidade de alertar os pais ou cui- também não tenha apresentado uma justificação. ${ }^{45}$ Por sua vez, a idade do pai não variou de forma significativa quanto a ter ou não TE. Não foram encontrados artigos nesta faixa etária que investigassem a associação, embora um estudo em crianças mais velhas (dos três aos cinco anos) também não tenha constatado a mes$\mathrm{ma}^{34}$

A associação do TE da criança com a escolaridade da mãe não é consensual na literatura, tendo sido verificada uma não associação na maioria dos estudos internacionais encontrados, ${ }^{5,35,41}$ à semelhança dos nossos resultados. Noutros estudos ${ }^{27,29}$ foi encontrada uma associação negativa, ou seja, mães com uma escolaridade superior (acima do $12^{\circ}$ ano de escolaridade) apresentavam filhos com menor TE. Em relação à escolaridade do pai, a não correlação com o TE verificada neste estudo foi concordante com a literatura. ${ }^{27}$

Também em conformidade com a literatura, ${ }^{5}$ não houve correlação entre o TE e o local/pessoa com que a criança passava a maior parte do dia [parental versus não parental (infantário, avós, outros)], embora este resultado não seja consensual. ${ }^{27,39} \mathrm{~A}$ ausência de correla- 
ção com o nível socioeconómico do agregado familiar foi concordante com a avaliação do nível socioeconómico em vários estudos. ${ }^{5,27,35,47}$ Contudo, alguns verificaram uma associação negativa, estando um nível socioeconómico mais baixo associado a um maior TE. ${ }^{29,37,44,48}$ De ressalvar que a forma de medir este nível socioeconómico variou entre eles.

A maioria dos pais/cuidadores das crianças referiu não ter sido informada acerca do TE adequado à faixa etária em questão, embora a resposta afirmativa não tenha sido correlacionada a não ter TE. De salientar que não foi avaliado o conhecimento das recomendações, apesar de ter sido reportada na literatura uma resposta correta em apenas $37 \%$ dos pais de crianças com idade inferior a dois anos. ${ }^{44}$

A taxa de resposta abaixo do esperado foi uma das limitações deste estudo, que compromete a representatividade da amostra. Esta deveu-se principalmente à falta de adesão dos participantes, causada provavelmente pela não receção do questionário por carta, pelo esquecimento da sua devolução, desinteresse ou não compreensão dos mesmos e limitação do tempo para a colheita de dados. Neste sentido, algumas das formas para colmatar esta falha seriam alterar a metodologia de distribuição dos questionários e alargar o prazo da colheita dos mesmos.

Existem ainda alguns vieses a considerar neste estudo, nomeadamente o viés de memória dos pais relativamente ao TE da criança e o seu desconhecimento dos hábitos de TE nas crianças que passam a maior parte do dia sob guarda não parental. Apesar de o questionário ter sido submetido a um processo de cognitive debriefing, o recurso a um questionário não validado pode também resultar num viés de medição, não garantindo a tradução correta do conhecimento que se pretendeu avaliar.

A nível nacional têm sido realizados cada vez mais estudos acerca dos hábitos de TE na idade pediátrica. Contudo, neste momento, são escassos aqueles que analisaram separadamente os dados de crianças com idade inferior aos 18-24 meses, em que é desaconselhado qualquer TE, à exceção da videochamada. ${ }^{1,28}$ Neste sentido, os autores deste estudo consideram-no uma mais-valia, na medida em que permitiu tirar conclusões mais fidedignas para este grupo, dada a diferença de recomendações entre as faixas etárias, re- lembrando a importância de reforçar as recomendações atuais de TE.

\section{CONCLUSÃO}

O TE na infância e o tempo diário de exposição são problemas crescentes, dados os riscos comprovadamente associados e a evidência limitada de benefício antes dos dois anos de idade. Contrariamente às recomendações atuais, as crianças com idade inferior a 18 meses estão a ser expostas a ecrãs. Segundo o verificado neste estudo, a maioria das características familiares não influencia os hábitos de consumo de TE nestas crianças. Nesse sentido, a elaboração de estratégias de intervenção generalizadas, nomeadamente a capacitação dos cuidadores acerca do TE adequado à idade da criança, deve ser incentivada.

A vigilância em consulta de Saúde Infantil permite uma abordagem dos pais de crianças numa idade precoce, sensibilizando-os para a importância de evitar qualquer TE em idades inferiores aos dois anos, à exceção da videochamada. Dada a elevada frequência recomendada de consultas programadas nestas idades, ${ }^{49}$ a informação pode ser reforçada com maior regularidade. A inclusão das recomendações atuais acerca do TE adequado a cada faixa etária no Programa Nacional de Saúde Infantil e Juvenil, da Direção-Geral da Saúde, poderá reforçar a importância da abordagem desta questão na consulta.

\section{REFERÊNCIAS BIBLIOGRÁFICAS}

1. Canadian Pediatric Society, Digital Health Task Force. Screen time and young children: promoting health and development in a digital world. Paediatr Child Health. 2017;22(8):461-77.

2. Common Sense Media. Zero to eight: children's media use in America 2013 [homepage]. Common Sense Media; 2015. Available from: https://www.commonsensemedia.org/zero-to-eight-2013-infographic

3. Fenstermacher SK, Barr R, Salerno K, Garcia A, Shwery CE, Calvert SL, et al. Infant-directed media: an analysis of product information and claims. Infant Child Dev. 2010;19(6):557-76.

4. Rideout $\mathrm{V}$. Parents, children \& media: a Kaiser Family Foundation survey [Internet]. Kaiser Family Foundation; 2007. Available from: http://files.eric.ed.gov/fulltext/ED542901.pdf

5. Zimmerman FJ, Christakis DA, Meltzoff AN. Television and DVD/video viewing in children younger than 2 years. Arch Pediatr Adolesc Med. 2007;161(5):473-9.

6. Cheung $\mathrm{CH}$, Bedford R, Saez-DeUrabain IR, Karmiloff-Smith A, Smith $T$ J. Daily touchscreen use in infants and toddlers is associated with reduced sleep and delayed sleep onset. Sci Rep. 2017;7:46104.

7. Mozafarian N, Motlagh ME, Heshmat R, Karimi S, Mansourian M, Mo- 
hebpour $F$, et al. Factors associated with screen time in Iranian children and adolescents: the CASPIAN-IV study. Int J Prev Med. 2017;8:31.

8. Barr R. Memory constraints on infant learning from picture books, television, and touchscreens. Child Dev Perspect. 2013;7(4):205-10.

9. Chassiakos YR, Radesky J, Christakis D, Moreno MA, Cross C. Children and adolescents and digital media. Pediatrics. 2016;138(5):e20162593.

10. Tomopoulos S, Dreyer BP, Berkule S, Fierman AH, Brockmeyer C, Mendelsohn AL. Infant media exposure and toddler development. Arch Pediatr Adolesc Med. 2010;164(12):1105-11.

11. Anderson DR, Kirkorian HL. Media and cognitive development. In: Lerner RM, editor. Handbook of child psychology and developmental science (Vol. 2). 7th ed. Wiley; 2015. chapter 22.

12. Zimmerman FJ, Christakis DA. Children's television viewing and cognitive outcomes: a longitudinal analysis of national data. Arch Pediatr Adolesc Med. 2005;159(7):619-25.

13. Chonchaiya W, Pruksananonda C. Television viewing associates with delayed language development. Acta Paediatr. 2008;97(7):977-82.

14. Byeon $\mathrm{H}$, Hong $\mathrm{S}$. Relationship between television viewing and language delay in toddlers: evidence from a Korea national cross-sectional survey. PLoS One. 2015;10(3):e0120663.

15. Lin LY, Cherng RJ, Chen YJ, Chen YJ, Yang HM. Effects of television exposure on developmental skills among young children. Infant Behav Dev. 2015;38:20-6.

16. Zimmerman FJ, Christakis DA, Meltzoff AN. Associations between media viewing and language development in children under age 2 years. J Pediatr. 2007;151(4):364-8.

17. Hinkley T, Verbestel V, Ahrens W, Lissner L, Molnár D, Moreno LA, et al. Early childhood electronic media use as a predictor of poorer well-being: a prospective cohort study. JAMA Pediatr. 2014;168(5):485-92.

18. Conners-Burrow NA, McKelvey LM, Fussell JJ. Social outcomes associated with media viewing habits of low-income preschool children. Early Educ Dev. 2011;22(2):256-73.

19. Tremblay MS, LeBlancAG, Kho ME, Saunders TJ, Larouche R, Colley RC, et al. Systematic review of sedentary behaviour and health indicators in school-aged children and youth. Int J Behav Nutr Phys Act. 2011;8:98.

20. Prentice-Dunn H, Prentice-Dunn S. Physical activity, sedentary behavior, and childhood obesity: a review of cross-sectional studies. Psychol Health Med. 2012;17(3):255-73.

21. Väistö J, Eloranta AM, Viitasalo A, Tompuri T, Lintu N, Karjalainen P, et al. Physical activity and sedentary behaviour in relation to cardiometabolic risk in children: cross-sectional findings from the Physical Activity and Nutrition in Children (PANIC) Study. Int J Behav Nutr Phys Act. 2014;11:55.

22. Grøntved A, Ried-Larsen M, Møller NC, Kristensen PL, Wedderkopp N, Froberg K, et al. Youth screen-time behaviour is associated with cardiovascular risk in young adulthood: the European Youth Heart Study. Eur J Prev Cardiol. 2014;21(1):49-56.

23. Kang HT, Lee HR, Shim JY, Shin YH, Park BJ, Lee YJ. Association between screen time and metabolic syndrome in children and adolescents in Korea: the 2005 Korean National Health and Nutrition Examination Survey. Diabetes Res Clin Pract. 2010;89(1):72-8.

24. Hardy LL, Denney-Wilson E, Thrift AP, Okely AD, Baur LA. Screen time and metabolic risk factors among adolescents. Arch Pediatr Adolesc Med. 2010;164(7):643-9.
25. Biddle SJ, Pearson N, Ross GM, Braithwaite R. Tracking of sedentary behaviours of young people: a systematic review. Prev Med. 2010;51(5): 345-51.

26. Stierlin AS, De Lepeleere S, Cardon G, Dargent-Molina P, Hoffmann B, Murphy $\mathrm{MH}$, et al. A systematic review of determinants of sedentary behaviour in youth: a DEDIPAC-study. Int J Behav Nutr Phys Act. 2015;12:133.

27. Certain LK, Kahn RS. Prevalence, correlates, and trajectory of television viewing among infants and toddlers. Pediatrics. 2002;109(4):634-42.

28. Council on Communications and Media. Media and young minds. Pediatrics. 2016;138(5):e20162591.

29. Schmidt ME, Rich M, Rifas-Shiman SL, Oken E, Taveras EM. Television viewing in infancy and child cognition at 3 years of age in a US cohort. Pediatrics. 2009;123(3):e370-5.

30. Anderson DR, Pempek TA. Television and very young children. Am Behav Sci. 2005;48(5):505-22.

31. Brown A. Media use by children younger than 2 years. Pediatrics. 2011;128(5):1040-5.

32. Dias A, Almeida C, Lobo AL, Veiga G. Os média e as crianças lá de casa... [Media and children at home...]. Saúde Infant. 2013;35(2):55-60. Portuguese

33. Tandon PS, Zhou C, Lozano P, Christakis DA. Preschoolers' total daily screen time at home and by type of child care. J Pediatr. 2011;158(2):297-300.

34. Downing KL, Hinkley T, Salmon J, Hnatiuk JA, Hesketh KD. Do the correlates of screen time and sedentary time differ in preschool children? BMC Public Health. 2017;17:285.

35. Vandewater EA, Rideout VJ, Wartella EA, Huang X, Lee JH, Shim MS. Digital childhood: electronic media and technology use among infants, toddlers, and preschoolers. Pediatrics. 2007;119(5):e1006-15.

36. Anand S, Krosnick JA. Demographic predictors of media use among infants, toddlers, and preschoolers. Am Behav Sci. 2005;48(5):539-61.

37. Dalzell VP, Msall ME, High PC. Parental attitudes of television and videocassette viewing of children aged birth to 36 months. J Dev Behav Pediatr. 2000;21(5):390.

38. McMillan R, Mclsaac M, Janssen I. Family structure as a predictor of screen time among youth. PeerJ. 2015;3:e1048.

39. Matarma T, Koski P, Löyttyniemi E, Lagström H. The factors associated with toddlers' screen time change in the STEPS Study: a two-year follow-up. Prev Med. 2016;84:27-33.

40. Kourlaba G, Kondaki K, Liarigkovinos T, Manios Y. Factors associated with television viewing time in toddlers and preschoolers in Greece: the GENESIS study. J Public Health (Oxf). 2009;31(2):222-30.

41. Thompson AL, Adair LS, Bentley ME. Maternal characteristics and perception of temperament associated with infant TV exposure. Pediatrics. 2013;131(2):e390-7.

42. Garcia-Continente X, Pérez-Giménez A, Espelt A, Nebot Adell M. Factors associated with media use among adolescents: a multilevel approach. Eur J Public Health. 2014;24(1):5-10.

43. Dennison BA, Erb TA, Jenkins PL. Television viewing and television in bedroom associated with overweight risk among low-income preschool children. Pediatrics. 2002;109(6):1028-35.

44. Patraquim C, Ferreira S, Martins H, Mourão H, Gomes P, Martins S. As crianças e a exposição aos media [Children and media exposure]. Nas- 
cer Crescer. 2015;24 Suppl 2:S11. Portuguese

45. Masur EF, Flynn V. Infant and mother-infant play and the presence of the television. J Appl Dev Psychol. 2008;29(1):76-83.

46. Laranjeira C, Vidal F, Peres S. Televisao, videojogos e internet: habitos de consumo [Television, videogames and internet: consumption habits]. Saúde Infant. 2008;30(3):98-101. Portuguese

47. Barr R, Danziger C, Hilliard M, Andolina C, Ruskis J. Amount, content and context of infant media exposure: a parental questionnaire and diary analysis. Int J Early Years Educ. 2010;18(2):107-22.

48. Thompson DA, Sibinga EM, Jennings JM, Bair-Merritt MH, Christakis DA. Television viewing by young Hispanic children: evidence of heterogeneity. Arch Pediatr Adolesc Med. 2010;164(2):174-9.
49. Direção-Geral da Saúde. Programa nacional de saude infantil e juvenil: norma n. ${ }^{\circ}$ 010/2013, de 31/05/2013. Lisboa: DGS; 2013.

\section{CONFLITO DE INTERESSES}

Os autores declaram não possuir quaisquer tipos de conflitos de interesse.

\section{ENDEREÇO PARA CORRESPONDÊNCIA}

Cátia Palha

E-mail: catiapalha@gmail.com

https://orcid.org/0000-0002-3194-8756

Recebido em 03-12-2018

Aceite para publicação em 27-09-2019

\section{ABSTRACT}

\section{INFLUENCE OF FAMILY CHARACTERISTICS ON SCREEN TIME USE IN CHILDREN UNDER 18 MONTHS OLD}

Aim: Assess family characteristics associated with screen time (ST) use in children aged 0 to 18 months.

Study: Descriptive and analytic cross-sectional study.

Place: USF Além D'Ouro, USF Camélias, USF Novos Rumos, USF Terras de Santa Maria, USF Vale do Vouga and UCSP de Crestuma.

Population: Children aged 0 to 18 months, enrolled in six health centers.

Methods: The authors developed a questionnaire about children's ST and a brief characterization of the family, with a voluntary and confidential response. Statistical analysis was performed using the Statistical Package for the Social Sciences - SPSS ${ }^{\circledR}$ program. Statistical significance was considered for $p$-values $<0.05$.

Results: Out of the 142 questionnaires returned (response rate 42.5\%), 137 were included. The median age was 12 months and $50.4 \%$ were male. The average father's age was 34 years and the mother's 33 . About $83 \%$ of the children belonged to nuclear families and 31.4\% were under the care of grandparents or in a nursery. Eighty-one percent of the 137 analyzed children had ST and of these, more than half had their first exposure before or at six months of age. There was no difference in duration of ST between the week and the weekends ( $71.2 \%$ and $73 \%$ of children with exposure, respectively). We found a statistically significant correlation between ST and child's age and mother's age, where children of mothers aged 39 or over were exposed to ST later.

Conclusion: This study showed unsuitable ST habits far from current age recommendations results consistent with national and international studies. Global intervention strategies, specifically training of caregivers about the adequate ST to child age, should be encouraged.

Keywords: Screen time; Infant; Family. 